\title{
Análisis de la atención de los usuarios en el área de hospitalización y su relación con un trato humanizado*
}

\author{
Analysis of the attention of the users in the area of \\ hospitalization and its relationship with humanized \\ treatment
}

DOLORES BARRIOS VILLALBA ${ }^{1}$

ClaRA SIERRA GARCÍA ${ }^{2}$

OMAIRA TORRES SANABRIA ${ }^{3}$

\footnotetext{
* Artículo producto de la investigación para la tesis de maestría titulada: "Análisis de la atención de los usuarios en el área de hospitalización y su relación con un trato humanizado”, Universidad Simón Bolívar, 2015

1 Magíster en Auditoría y sistemas de calidad en servicio de salud. dbarrios@unisimonbolivar.edu.co.

2 Odontóloga, Especialista en Sistemas de Garantía de la Calidad y Audioría de Servicios de Salud, Maestría en Auditoría y calidad de servicios de salud. cgarcia@unisimonbolivar.edu.co.

3 Magíster en Auditoría y sistemas de calidad en servicio de salud. otorres@unisimonbolivar.edu.co.
} 


\section{RESUMEN}

En este trabajo de investigación queda plasmada la información recogida al evaluar la atención en el área de Hospitalización y su relación con un trato humanizado en una E.S.E., de $2^{\circ}$. Nivel de atención en Salud del distrito de Barranquilla, factor que ha cobrado gran protagonismo en el tiempo reciente por las exigencias a nivel global en cuanto a calidad y oportunidad a los usuarios de los servicios de salud. Con el fin de satisfacer las necesidades de los pacientes, se hace necesario identificar cúal es la percepción de la atención que ellos tienen al momento de ingresar en el área de hospitalización, y para este propósito se realizará un ejercicio de reflexión en el Hospital Nazaret IPS Universitaria, en el barrio Cevillar de la ciudad de Barranquilla.

Dentro de los Derechos fundamentales que tienen los colombianos, se enuncian el Derecho a la Vida y la Salud, contemplado en la Constitución Política colombiana, derechos que debe garantizar el Estado, desde el Ministerio de Salud y Protección Social, ofreciendo a los usuarios del Sistema de Seguridad Social en Salud, el cumplimiento desde la atención primaria, hasta la hospitalización, si el caso lo requiere. Por ende, la calidad en la atención al paciente es un factor de mucha preocupación para los responsables de la prestación de los servicios de salud, ante la solicitud de los pacientes que llegan a las entidades de salud. Esta situación amerita la evaluación constante de los funcionarios que prestan estos servicios implementando estrategias para calificar la calidad en la atención al usuario.

Palabras clave: salud, humanización, hospitalización, pacientes, calidad.

\section{ABSTRACT}

In this research, the information gathered in the evaluation of care in the area of hospitalization and its relation to a humanized treatment in a sec- 
ond-level hospital are captured. Level of care in Health of the District of Barranquilla, factor that has gained great prominence in the recent time by the demands at global level in quality and opportunity to the users of the health services. In order to satisfy the patients' needs, it is necessary to identify the perception of the care they have, when entering the hospitalization area, and for this purpose a reflection exercise will be carried out at the Hospital Nazaret IPS University, in the Cevillar neighborhood of the city of Barranquilla.

Within the Fundamental Rights of Colombians, the right to life and health, as set forth in the Colombian Constitution, are enunciated. These rights must be guaranteed by the State, from the Ministry of Health and Social Protection, Social Security System in Health, compliance from primary care, to hospitalization, if the case requires it. Therefore, the quality of patient care is a factor of great concern for those responsible for the delivery of health services, at the request of patients arriving at health institutions. This situation warrants the constant evaluation of health service providers, implementing strategies to qualify the quality of care for the user.

Key words: health, humanization, hospitalization, patients, quality.

\section{INTRODUCCIÓN}

En la prestación de servicios de atención en el campo de la salud, se ve actualmente una preocupación latente porque sea humanizado a los usuarios y vinculados a las entidades de salud. Este factor viene convirtiéndose en una ventaja competitiva que genera calidad y compromiso, tanto en las entidades como los profesionales que vienen prestando el servicio.

Mundialmente, la Salud es un derecho fundamental para el desarrollo de todo ser humano, exigiendo del sistema de atención en este sentido, un 
alto compromiso para el direccionamiento de las mejoras pertinentes y estrategias adecuadas según las necesidades del entorno. La Constitución de Colombia de 1991, enuncia que la seguridad social es un servicio público obligatorio direccionado y controlado por el Estado. La Ley 100 de 1993 realiza cambios en el servicio de salud, dándole la importancia a los pacientes, teniendo en cuenta los cambios administrativos, financieros y de contratación que la actualidad de hoy demanda para responder a los distintos desafíos.

Estos cambios han permitido un mejor servicio en la atención de los usuarios en las entidades de salud del país habiéndose legislado en el Decreto 2309 de 2002 el Sistema Obligatorio de Garantía de Calidad, de la Atención de Salud del Sistema General de Seguridad Social, cumpliendo así el objetivo de sensibilizar y humanizar al personal que presta sus servicios en las entidades de salud.

Este trabajo de investigación, realizado en la E.S.E. Hospital Nazaret IPS Universitaria del Distrito de Barranquilla, tiene la intención de identificar cuál es la percepción que tienen los pacientes sobre las fallas y beneficios que tienen los funcionarios en la humanización de la atención a los usuarios, estableciendo las fallas que tienen los empleados en el logro de este objetivo y las posibles mejoras que se pueden incorporar.

\section{TRATO HUMANO EN LA SALUD, UNA NECESIDAD LATENTE}

Los aspectos médicos y curativos han tenido por siempre en la sociedad una influencia histórica, lo que da al servicio de salud un carácter de especial atención al interior de cualquier país, población o colectivo.

Poder generar bienestar a partir de la práctica médica, se ha constituido para muchos gobiernos en una bandera de trabajo, que garantiza no solo 
el desarrollo humano, sino también la elevación del nivel de vida y la percepción de felicidad en este sentido. El servicio clínico u hospitalario se visiona como un conjunto de procesos que, al estar en equilibrio, brindan a todo individuo la sensación de seguridad y estabilidad que necesita para realizar casi cualquier actividad de manera solvente y dinámica (Appleby \& Camacho, 2014).

No obstante, es indispensable que el servicio de salud no se limite solo a la prestación de procedimientos o protocolos, además, debe integrar un componente humano que transmita a toda persona, en un proceso de enfermedad, tratamiento o recuperación, un sentido de calidez, importancia o representación, que contribuya desde lo humano a su restablecimiento en el ámbito de la salud.

La globalización socio-económica, que ha venido transformando y haciendo evolucionar muchos aspectos organizacionales ha llegado también al sector salud y demanda del mismo, que los profesionales al servicio de los usuarios, no se conformen con la prestación simple o plana del servicio, sino que incorporen dentro de su quehacer cotidiano, prácticas apropiadas para que el paciente o usuario se sienta como lo que es, el centro del sistema de salud, de cualquier comunidad o población (Arcos, E, et al, 2007; Barrera, Pinto \& Sánchez, 2010; Ceballos, 2010).

Al reconocer al usuario como actor principal del sistema de salud, se le está devolviendo un sitial que se ha había desdibujado las décadas recientes, donde aspectos como la incorporación de nuevas tecnologías, fueron enfriando o automatizando la atención en salud, dejando de lado un aspecto fundamental que se conoce como trato humano.

$\mathrm{Al}$ describir el trato humanizado, humanización o gestión de salud humanizada, como la han denominado diversos autores, se deben tener en cuen- 
ta los siguientes factores (Gil, 2002):

- El ser humano debe ser valorado como un individuo con emociones que requiere tanto de la atención científica como personal.

- El profesional de servicios médicos debe ser una persona enfocada al servicio, de lo contrario no brindará el trato humano que requieren los usuarios o pacientes.

- Las instituciones donde se presta el servicio salud deben trabajar por proveer al personal encargado de la prestación de los servicios, herramientas, estímulos e incentivos para que esta labor se preste siempre bajo las mejores condiciones.

El servicio de tipo humanista o humanizado, ha sido descrito como aquel donde el usuario es escuchado, es visible y donde sus emociones son tomadas en cuenta como parte fundamental del proceso de recuperación. Brindar al usuario la oportunidad de expresarse y a través de este proceso permitir que sea parte de su proceso de curación o tratamiento se considera hoy día algo esencial para la recuperación, especialmente cuando se está frente a enfermedades calamitosas, poco conocidas o de gran compromiso para el afectado.

El cuidado del ser humano debe desarrollarse bajo un ambiente de reciprocidad, donde la calidad y calidez sean auténticas, pues es la única forma que este pueda alcanzar un estado óptimo de bienestar. Los profesionales médicos están llamados a prestar al paciente un cúmulo de servicios que van más allá de los protocolos y procedimiento; investigadores en el tema hablan de la importancia de lograr el equilibrio entre el cuerpo y la mente, pues es ahí donde está la clave para obtener resultados asombrosos en condiciones adversas (D’Empaire, 2010; Cárdenas, Melengue, Pinilla, Carrillo \& Chaparro, 2010). 
Por lo anterior, el profesional médico, cualquiera sea su nivel o campo de ejercicio, es considerado un agente humanitario que proporciona bienestar de tipo moral y se considera un transformador de realidades sociales que afectan tanto al individuo como a su círculo familiar. Cuando hablamos de profesionales médicos, no solo se hace referencia a los doctores, especialistas o cirujanos, intervienen también todos los auxiliares, asistentes, administrativos y demás actores involucrados en la prestación del servicio al usuario (Sánchez, 2012).

Lo precedente, genera entonces otros interrogantes sobre el papel protagónico que debe jugar cada uno de los actores del proceso, ya que usualmente se le da mayor importancia al trabajo del personal que atiende de manera directa como médicos y enfermeras y se deja de hacer seguimiento, por ejemplo, al trabajo que realizan administrativos en facturación, dispensación de medicamentos y trámites de citas médicas. Todos estos procesos que hacen parte del entorno del servicio de salud también pueden contribuir con la armonía y estabilidad del paciente, ya que, al sentirse parte del proceso e importante para todos los integrantes del sistema, desarrollará nuevas dinámicas que le empujarán a una recuperación más ponderada y destacable (Geiger, Heermann \& Eilers, 2005; Sánchez, 2014).

Lo anterior es tan importante que muchas IPS a nivel de Latinoamérica, han iniciado programas de cumplimiento y desempeño, donde estimulan a todo el personal asociado a la prestación de los servicios, para cumplir indicadores donde el trato cálido y amable, es medido a través de encuestas permanentes que los usuarios realizan una vez finaliza el período de atención. Estas entidades miden tanto la asignación de una cita médica, entregas de medicamentos y explicación de procesos complementarios al servicio de atención directa (Grau, Flichtentrei, Suñer, Prats \& Braga, 2009; Horvath, et al, 2005; Mason \& Harrison, 2008). 
Estas mediciones han procreado en los funcionarios, un ambiente de compromiso y derivado progresivamente, en la puesta en marcha de mejoras, que los mismos funcionarios y usuarios van sugiriendo para mejorar la plataforma de servicios. Esta continua comunicación e interacción, se ha convertido entonces, en una fuente de ideas y creatividad, debido a que cada vez que se presenta una inconsistencia o inconformidad, el ambiente abierto y amable permite que las partes se integren en el proceso de solución.

Todo lo expuesto hace evidente que la única forma de lograr un trato humanizado, compromete la integración de todos los componentes y actores del proceso, pues solo de esta forma se podrán insertar en los momentos precisos los cambios necesarios para cada situación al interior del sistema de salud. Esto hace suponer entonces que cada sistema de salud opera de forma particular, y aunque existen similitudes entre uno y otros, la mejor fórmula para alcanzar el escenario de trato humano que se busca es observar las condiciones y particularidades de cada entorno, población o comunidad, para así dar paso al escenario objetivo en cada sistema (Lau, et al, 2009; Mercado \& Hernández, 2007).

\section{METODOLOGÍA}

El presente estudio ha permitido describir de modo sistémico la situación del servicio de salud prestado a los pacientes que llegan a las diferentes entidades prestadoras del servicio en Barranquilla. Ha permitido realizar un tipo de investigación descriptiva, en la cual predomina la actitud tanto del paciente como del profesional de la salud, permitiendo recolectar los datos en forma auténtica y veraz. El personal para este estudio se escogió en el Hospital Nazaret IPS Universitaria en el barrio Cevillar, donde se utiliza un muestreo que brinda a todos los pacientes las mismas condiciones de selección; esto se logró en forma aleatoria en las diferentes áreas específicas como son Urgencias y Hospitalización, escogiendo 75 pacientes de cada una de las áreas para un total de 150 usuarios del servicio de atención. 
Se obtuvo en las Fuentes Primarias información de manera directa a través de encuestas confiables, utilizando las historias clínicas, con el fin de evaluar la calidad en la prestación del servicio y el grado de humanización del personal que interviene en la atención a los pacientes.

\section{RESULTADOS}

En los resultados se observan detalles más amplios de diversas variables que es importante considerar.

Tabla 1

Nivel de usuarios que solicitan servicios en IPS

\begin{tabular}{ccc}
\hline Ítem & Frecuencia & Porcentaje \\
\hline Medio & 40 & $26,6 \%$ \\
Bajo & 110 & $73,3 \%$ \\
Total & 150 & $100 \%$ \\
\hline \multicolumn{2}{c}{ Fuente: Encuesta aplicada a usuarios del servicio de atención de la ESE }
\end{tabular}
Hospital de Nazareth

Los resultados dejan observar que, de los usuarios encuestados, pertenecen a estrato socio-económico Bajo (72,9 \%), y el resto a estrato Medio $(27,1 \%)$, permitiendo así que la población de pocos recursos sea la más afectada. Con referencia al tema de acceso a medicamentos de manera regular y oportuna, se detallan los resultados siguientes.

\section{Tabla 2}

Acceso a medicamentos

\begin{tabular}{ccc}
\hline Ítem & Frecuencia & Porcentaje \\
\hline Regular & 120 & $80 \%$ \\
Malo & 30 & $20 \%$ \\
Total & 70 & $100 \%$ \\
\hline $\begin{array}{l}\text { Fuente: Encuesta aplicada a usuarios del servicio de atención de la ESE } \\
\text { Hospital de Nazareth }\end{array}$
\end{tabular}

Gráficamente podemos observar que el acceso regular a los medicamentos llega a un $80 \%$, entre tanto, un $20 \%$ califica como mal servicio la adquisi- 
ción de los mismos; no existe un servicio a satisfacción de los pacientes, ya que no predomina un servicio calificado como bueno.

En la siguiente instancia se evalúa uno de los aspectos neurálgicos. Es el de la hospitalización, ver Tabla 3.

Tabla 3

Valoración del servicio de hospitalización

\begin{tabular}{lcc}
\hline \multicolumn{1}{c}{ ITEM } & Frecuencia & Porcentaje \\
\hline Parto y postparto & 90 & $60 \%$ \\
Enfermedades respiratorias & 20 & $13,3 \%$ \\
Enfermedades digestivas & 15 & $10 \%$ \\
Enfermedades cardiovasculares & 8 & $5,3 \%$ \\
Tumores & 4 & $2,6 \%$ \\
Heridas y contusiones & 4 & $2,6 \%$ \\
Enfermedades genitourinarias & 3 & $2 \%$ \\
Fiebre reumática & 3 & $2 \%$ \\
Otras & 3 & $2 \%$ \\
Total & 150 & $100 \%$ \\
\hline
\end{tabular}

Fuente: Encuesta aplicada a usuarios del servicio de atención de la ESE Hospital de Nazareth

La mayor prevalencia en hospitalización se encuentra en los partos que alcanza un $90 \%$, seguido de enfermedades respiratorias con un $20 \%$, y enfermedades digestivas un $15 \%$; estos tres ítem son los de mayor impacto y representación en el cuadro general.

A continuación se analiza uno de los aspectos más relevantes para el usuario y corresponde a la asignación de citas. En la Tabla 4, se muestran los hallazgos.

\section{Tabla 4}

Tiempo para asignación de citas

\begin{tabular}{|c|c|c|}
\hline Ítem & Frecuencia & Porcentaje \\
\hline De 1 a 3 días & 75 & $50 \%$ \\
\hline De 4 a 7 días & 45 & $30 \%$ \\
Más de 15 días & 30 & $20 \%$ \\
\hline Total & 70 & $100 \%$ \\
\hline $\begin{array}{l}\text { Fuente: Encuesta aplicada a usuarios del servicio de atención de la ESE } \\
\text { Hospital de Nazareth }\end{array}$
\end{tabular}


En esta asignación podemos observar que la atención de las citas es buena, teniendo un $50 \%$ en menos de tres días, un $30 \%$ en menos de 7 días y solo un 20 \% sobre los 15 días. En el siguiente apartado se revisa el aspecto central que valora la atención o servicio y la percepción de este importante aspecto en cada paciente o usuario encuestado, ver tabla 5 .

\section{Tabla 5}

Percepción de la atención recibida (humanización)

\begin{tabular}{c|c|c|}
\hline Ítem & Frecuencia & Porcentaje \\
\hline Bueno & 76 & $50.6 \%$ \\
Adecuado & 44 & $29.3 \%$ \\
Regular & 30 & $20 \%$ \\
\hline Total & 70 & $100 \%$ \\
\hline Fuente: Encuesta aplicada a usuarios del servicio de atención de la ESE
\end{tabular}

Hospital de Nazareth

Los usuarios califican el servicio recibido de manera positiva, un 50,6 \% lo estima bueno, un 29,3 \% adecuado y solo un $20 \%$ lo valora regular.

\section{DISCUSIÓN}

Los resultados obtenidos permiten afirmar que el trabajo de humanización viene marcando pauta en esta IPS, y que los lineamientos implantados por la organización sí dejan huella en las personas o usuarios que acuden a recibir la atención necesaria en esta entidad. Seguir las disposiciones que desde el Ministerio de Salud y de la Protección Social se han sugerido viene arrojando buenos dividendos para esta institución, lo que beneficia no solo el ambiente en general, sino que además, deriva en un posicionamiento de la imagen, aspecto diferencial y muy crucial que toda entidad de salud debe tener en cuenta en el país (Nilsson, Öhman \& Söderberg, 2006).

Como han expuesto algunos autores, los cambios de hoy día son complejos, no obstante se pueden lograr buenos resultados si la disposición y motivación están al servicio del sector salud. El papel del recurso humano es esencial, ya que si los individuos que hacen parte de la planta de operación de las IPS, acogen de buena manera los mandatos y demás consideracio- 
nes para la atención del usuario, se pueden ponderar todas las líneas de atención, y áreas como urgencias y hospitalización pueden elevar su nivel, lo que beneficia de manera directa al usuario final (Milos, Larrain \& Simometti, 2009; OMS, 2014).

Por lo citado, las IPS están llamadas a implementar de manera pronta, todos los protocolos y demás planteamientos que exige el Estado, ya que de esta forma, se puede generar un gran impacto social en las ciudades, municipios y demás localidades de todo el territorio.

\section{CONCLUSIONES}

Al final de esta propuesta se puede enunciar que el estudio debe ser una punta de lanza para futuras investigaciones, ya que estos aspectos son susceptibles de estudios constantes y profundos para desarrollar planes de intervención oportunos que deriven en calidad de servicios para los usuarios. En el distrito de Barranquilla, lo precedente es fundamental, ya que el sector salud viene en un proceso de crecimiento y evolución que debe ser ponderado y atendido de manera prioritaria.

Igualmente, se puede afirmar que los objetivos esperados se obtuvieron determinando las variables que configuran la percepción actual del usuario y se logró también detallar, factores que a futuro pueden ser mejorados aún más, como la tramitación de citas médicas en ciertos casos. Es vital reconocer que la disposición del personal es crucial para alcanzar los objetivos esperados, por lo cual su bienestar y estabilidad deben ser revisados de forma constante para alcanzar resultados perdurables en este sentido.

\section{REFERENCIAS BIBLIOGRÁFICAS}

Appleby, C. \& Camacho, R. (2014). Retos y oportunidades: aportaciones de la Enfermera de Práctica Avanzada en la cronicidad. Aprendiendo de las experiencias. Enfermería Clínica, 24(1), 90-98.

Arcos, E., Poblete, J., Molina, I., Miranda, C., Zuñiga, Y., Fecci, E, et al. 
(2007). Perspectiva de género en la formación de profesionales de la salud: Una tarea pendiente. Rev. méd. Chile, 135(6), 706-717.

Barrera, L., Pinto, N. \& Sánchez, B. (2010). Capítulo 2: Situación del Cuidador Familiar. Caracterización de los Cuidadores en América Latina. En: Barrera L, Pinto N, Sánchez B. Cuidando a los Cuidadores. Bogotá: Universidad Nacional de Colombia.

Cárdenas, D., Melenge, B., Pinilla, J., Carrillo., G., Chaparro, L. (2010). Soporte social con el uso de las TIC para cuidadores de personas con enfermedad crónica: un estado del arte. Aquichán, 1O(3), 204-213.

Ceballos, P. (2010). Desde los ámbitos de enfermería, analizando el cuidado humanizado. Cienc. Enferm, 16(1), 31-35.

D’Empaire G. (2010). Calidad de Atención Médica y Principios Éticos. Acta bioeth, 16(2), 124-132.

Geiger, D., Heermann, J. \& Eilers J. (2005). Identification and validation of competencies for use in objective structured clinical examinations for lay caregivers. Cancer Nursing, 28(1), 54-61.

Gil, P. (2002). Influencia del género sobre el proceso de desarrollo del Síndrome de quemarse por el trabajo (burnout) en profesionales de enfermería. Psicología em Estudo, 7(1), 3-10.

Grau, A, Flichtentrei, D., Suñer, R., Prats, M. \& Braga F. (2009). Influencia de factores personales, profesionales y transnacionales en el síndrome de burnout en personal sanitario hispanoamericano y español. Rev. Esp. Salud Pública, 83, 215-230.

Horvath, K., Hurley, A., Duffy, M., Gauthier, M., Harvey, R., Trudeau, S. et al. (2005). Caregiver Competence to Prevent Home Injury to the Care Recipient with Dementia. Rehabilitation Nursing, 3o(Issue 5), 189-196.

Lau, D., Kasper J., Hauser, J., Berdes, C., Chang, C., Berman, R., et al. (2009). Family Caregiver Skills in Medication Management for Hospice Patients: A Qualitative study to Define a Construct. Journals of Gerontology Series B: Psychological Sciences \& Social Sciences, 64B (Issue 6), 799-807. 
Mason, B. \& Harrison, B. (2008). Telephone Interventions for Family Caregivers of Patients with Dementia What Are Best Nursing Practices? Feature, 22(6), 348-354.

Mercado, F. \& Hernández, E. (2007). Las enfermedades crónicas desde la mirada de los enfermos y los profesionales de la salud: un estudio cualitativo en México. Cad. Saúde Pública, 23(9): 2178-2186.

Nilsson, C., Öhman, M. \& Söderberg, S. (2006). Information and communication technology in supporting people with serious chronic illness living at home- an intervention study. Journal of Telemedicine and Telecare, 12, 198-202.

Milos, P., Larrain, A. \& Simometti, M. (2009). Categorización de Servicios de Enfermería: Propuesta para Asegurar una Atención de Calidad en Tiempos de Escasez de Enfermeras. Cienc. Enferm, 15(1), 17-24.

Organización Mundial de la Salud (OMS) (2015). Estadísticas sanitarias mundiales 2014. Disponible en: http://apps.who.int/iris/bitstream/10665/112817/1/WHO_HIS_HSI_14.1_spa.pdf?ua=1

Pesante, R. F. (2016). Percepción de atención humanizada y su relación con la calidad de atención en el servicio de radiodiagnóstico. UCV-SCIENTIA, 8(2), 124-131.

Romero Massa, E., Méndez, C., Maria, I., \& Moncada Serrano, A. (2016). Relationship between humanized care through nursing and hospitalization for patients. Hacia la Promoción de la Salud, 21(1), 26-36.

Sánchez C. (2014). Inmaculada Cronicidad y Complejidad: Nuevos Roles en Enfermería. Enfermeras de Práctica Avanzada y Paciente Crónico. Enfermería Clínica, 24(1), 79-89.

Sánchez, B. (2012). Dimensión espiritual del cuidado en situaciones de cronicidad y muerte [Internet]. [Consultado 2014 Nov 14]. Disponible en: http://www.bdigital.unal.edu.co/2050/1925/9789587611335.pdf.

Cómo citar este capítulo:

Barrios Villalba, D., Sierra García, C., \& Torres Sanabria, O. (2017). Análisis de la atención de los usuarios en el área de hospitalización y su relación con un trato humanizado. En J. Rodríguez López, M. Gómez Barbosa, D. Martínez Sierra, H. Hernández Palma, C. Sierra García, C. Beleño Agudelo, . . . J. Bermejo Urzola, Investigaciones y casos del sector salud de la región Caribe (pp.59-72). Barranquilla: Ediciones Universidad Simón Bolívar. 